\title{
ECS-induced amnesia and recovery of memory for classically conditioned fear in the rat
}

\author{
RALPH R. MILLER, CLIFFORD A. OTT, DAVID DANIEL, and ALVIN M. BERK \\ Brooklyn College of the City University of New York, Brooklyn, New York 11210
}

\begin{abstract}
Using escape from conditioned aversive stimuli as an indirect index of strength of classical conditioning, one-trial conditioning was demonstrated. Electroconvulsive shock immediately following the unconditioned stimulus (footshock) yielded amnesia for the conditioned associations, and a single "reminder" footshock given in an environment dissimilar to the training apparatus restored memory in both between- and within-subjects designs. These results are inconsistent with the view that recovery induced by a noncontingent reminder shock from experimental amnesia depends upon classically conditioned associations that survive treatment by amnestic agents.
\end{abstract}

Traditionally, the occurrence of experimental amnesia for information acquired immediately preceding electroconvulsive shock (ECS) has been interpreted as evidence supportive of the consolidation theory of memory (e.g., McGaugh, 1966). Recently, however, considerable evidence has been gathered which, without necessarily challenging consolidation theory per se, suggests that ECSinduced amnesia is due to impaired retrieval rather than the disruption of consolidation (Miller \& Springer, 1973). One of the major points supporting this alternative hypothesis is that ECS-induced amnesia appears to be reversible, a result which is inconsistent with the disrupted consolidation view of experimental amnesia, which hypothesizes that the loss of memory is permanent. While there is little evidence supporting the spontaneous recovery of memory after ECS, a number of manipulations have been identified that do result in a reduction of the observed behavioral deficit. Gold and King (1974) have suggested that this apparent recovery of memory is an artifact of learning that occurs during the restoration treatment and generalizes to the test apparatus. However, Miller and Springer (1974) have pointed out several inadequacies of this hypothesis in respect to the available data.

More recently, Schneider, Tyler, and Jinich (1974) have proposed another explanation of the apparent recovery from ECS-induced amnesia that is consistent with consolidation theory. Noting the frequent observation that classically conditioned associations appear to be relatively immune to the amnestic effects of ECS, they hypothesized that, in those studies in which the experimental animals receive more than one test trial, the apparatus cues on the first test trial act

This research was supported in part by U.S. Public Health Service Grant MH-19497 and by CUNY Faculty Research Award Program Grant RF10694. Reprint requests should be sent to Ralph R. Miller, Department of Psychology, Brooklyn College of CUNY, Brooklyn, New York 11210. as conditioned stimuli which evoke classically conditioned fear in the animal and to which the animal learns appropriate instrumental behavior on the same trial. Then, on a subsequent test trial, the apparent recovery of memory is really a manifestation of instrumental learning acquired on the preceding test trial. This hypothesis is intriguing; however, it fails to adequately explain a fairly large number of reported instances of recovery from experimental amnesia. First, a number of studies report recovery of memory in between-group paradigms in which each animal is given only a single test trial (e.g., Miller \& Springer, 1972). Secondly, restoration of appetitive memories following ECS has been reported (Miller, Ott, Berk, \& Springer, 1974), and it is not at all clear how the hypothesis of Schneider et al. would deal with this sort of data. Nevertheless, due to the significance of the recovery phenomenon, we felt that the proposal of Schneider et al. was worthy of further examination. The following experiment was designed as a test of their hypothesis.

While most researchers report a failure to obtain ECS-induced amnesia for classically conditioned associations, there are a number of studies in which amnesia for classical conditioning was obtained. Springer (1975) resolved this discrepancy by identifying a number of task and treatment parameters that determine when amnesia for classically conditioned associations will occur. The intent of the present study was to use a set of parameters at which amnesia could be obtained for classical conditioning and then to see if recovery of memory could be obtained. Such a result would be inconsistent with the hypothesis of Schneider et al. but consistent with the retrieval failure view of experimental amnesia.

\section{METHOD}

\section{Subjects and Apparatus}

The subjects were 50 male Sprague-Dawley-descended rats obtained from a commercial breeder (Carworth, New City, New 
Table 1

Treatments and Means of Individual Mean Escape Reciprocal Latencies

\begin{tabular}{|c|c|c|c|c|c|c|c|c|}
\hline \multirow[b]{2}{*}{ Group } & \multicolumn{8}{|c|}{ Treatment } \\
\hline & Day 1 & Day 2 & Day 3 & Day 4 & & Day 5 & & Day 6 \\
\hline ACQ & Adaptation & FS & & Test $\left(.615 \mathrm{sec}^{-1}\right)^{*}$ & & & & \\
\hline $\mathrm{AMN}$ & Adaptation & FS and ECS & & Test $\left(.144 \sec ^{-1}\right) \#$ & $<$ & Remind & $\begin{array}{l}\text { Retest } \\
\text { Retest }\end{array}$ & $\begin{array}{l}\left(.600 \sec ^{-1}\right)^{*} \\
\left(.188 \sec ^{-1}\right) \#\end{array}$ \\
\hline $\begin{array}{l}\text { REM } \\
\text { NC }\end{array}$ & $\begin{array}{l}\text { Adaptation } \\
\text { Adaptation }\end{array}$ & $\begin{array}{l}\text { FS and ECS } \\
\text { Noncontingent } \\
\text { FS and ECS }\end{array}$ & $\begin{array}{l}\text { Remind } \\
\text { Remind }\end{array}$ & $\begin{array}{l}\text { Test }\left(.524 \sec ^{-1}\right)^{*} \\
\text { Test }\left(.145 \sec ^{-1}\right) \#\end{array}$ & & & & \\
\hline
\end{tabular}

Note-Means designated * did not differ among themselves but were each significantly different (all ps $<.05)$ from each mean designated \# which in turn did not differ among themselves.

York). The animals weighed from 170 to $190 \mathrm{~g}$ on Day 1 of the study, were individually housed in a wire mesh cage, and were maintained on ad-lib water and $10 \mathrm{~g} /$ day of powdered Purina Lab Chow given approximately $1 \mathrm{~h}$ after being run on experimental days. The experiment was run in four balanced replications, except for the addition of a single animal to one of the groups in each of the two last replications.

The apparatus used for conditioning and testing was a two-compartment Plexiglas shuttlebox with an illuminated white compartment having a grid floor and a darkened black compartment having a plywood floor. The compartments, each measuring $24 \times 11 \times 14 \mathrm{~cm}$, were separated by a guillotine door and a 4-cm hurdle. The shuttlebox was equipped with counterweighted ECS earclip electrodes that permitted the animal complete freedom of movement. Also employed in this study were two enclosures quite dissimilar to the conditioning apparatus: a holding cage resembling the home cages and a metal enclosure measuring $15 \times 75 \times 75 \mathrm{~cm}$.

\section{Procedure}

The procedure, a modification of one developed by McAllister and McAllister (1968), is outlined in Table 1. All animals received $2 \mathrm{~min} /$ day of handling by the experimenter on each of the 4 days immediately prior to Day 1 of the study. On Day 1 , the animals were adapted to each compartment of the shuttlebox with the connecting door closed. Subjects spent $7.5 \mathrm{~min}$ in each compartment interrupted three times for $0.5 \mathrm{~min}$ of handling and 2 min of adaptation to the holding cage. Order of exposure to the black and white compartments was counterbalanced across animals. All subjects wore earclips for the first 2 min of adaptation in the white compartment only.

On Day 2, the training day, animals in Groups ACQ $(n=12)$, $\operatorname{AMN}(n=14)$, and REM $(n=12)$ were individually placed in the holding cage. After $30 \mathrm{sec}$, each of these animals had earclips affixed and was transferred to the white compartment of the shuttlebox with the intercompartmental door closed. Fifteen seconds later, a 4-mA footshock (FS) of $0.5 \mathrm{sec}$ duration was administered. Groups AMN and REM received a $60-\mathrm{mA}$ ECS of $0.3 \mathrm{sec}$ duration immediately following FS offset, and were then returned to the home cage. Subjects in Group ACQ were left in the white compartment for $10 \mathrm{sec}$ following FS offset, and then were placed in the holding cage for an additional $30 \mathrm{sec}$ prior to return to the home cage. Subjects in Group NC $(n=12)$ received the identical FS and ECS as subjects in Groups AMN and REM but in the presence of different apparatus cues; hence, the NC animals constituted a noncontingent group in the classical conditioning sense in that their exposure to the US was in the absence of the CS. Specifically, members of Group NC were placed in the hold ing cage for $30 \mathrm{sec}$, then had earclips attached and were placed in the metal enclosure. Fifteen seconds later, each subject received FS and ECS and then was returned to the home cage.

On Day 3, subjects in Groups REM and NC were placed in the holding cage for $30 \mathrm{sec}$, after which they were transferred to the metal enclosure. Fifteen seconds later, each animal received a 4-mA FS of $2.0 \mathrm{sec}$ duration. The duration of this "reminder" FS, greater than the training FS, had been determined in pilot studies to be the most effective "reminder" FS duration, given all the other treatment parameters. Ten seconds after FS offset, each animal was transferred to the holding cage for an additional $30 \mathrm{sec}$ before being returned to the home cage.

On Day 4, the test day, all subjects were given the opportunity to learn to "instrumentally" escape from the apparatus cues of the white compartment into the black compartment. There was no shock administered on this day. Specifically, each animal received 25 hurdle-jumping trials, each preceded by $30 \mathrm{sec}$ in the holding cage. A hurdle-jumping trial consisted of placing the animal in the white compartment facing away from the intercompartmental door, waiting $10 \mathrm{sec}$, opening the door between the two compartments, and permitting the animal a maximum of $60 \mathrm{sec}$ to jump over the hurdle. Latencies were measured from the time the intercompartmental door was opened to the time that a photocell beam in the black compartment $15 \mathrm{~cm}$ from the hurdle was interrupted. Ten seconds after entering the black compartment, each animal was transferred to the holding cage to begin the 30 -sec ITI. It should be noted that, while the behavior observed on the test trials was instrumental, no relevant instrumental training had been given on the training day in that the intercompartmental door had not been open. Pilot studies indicated that rats that never received FS would shuttle once they noticed the open doorway, but would never attain the speed of shocked animals. Behaviorally, these nonshocked animals were very similar to the NC animals in the present study.

On the third and fourth replications, the AMN animals were divided into two subgroups counterbalanced for individual mean Day 4 performance. On Day 5, one of these subgroups was given a "reminder" FS identical to the treatment of REM and NC animals on Day 3. On Day 6, animals in both of these AMN subgroups were given 25 additional hurdle-jumping trials identical to those administered on Day 4.

\section{RESULTS}

Between-groups comparisons of Day 4 data indicated that Groups ACQ and REM exhibited shorter latencies than Groups AMN and NC. Within-groups comparisons between Day 4 and Day 6 performances yielded reduced latencies only in the AMN subgroup that received FS on Day 5.

To increase the normality of the data, all latencies were transformed to reciprocals. Mean reciprocal latencies over the 25 hurdle-jumping trials were then calculated for each animal. Group means of these scores are indicated in Table 1. All statistical tests reported are two-tailed. An analysis of variance of Day 4 scores yielded a significant treatment effect, 
$\mathrm{F}(3,34)=31.77, \mathrm{p}<.001$, and $\mathrm{a}$ significant replication effect, $F(3,34)=2.97, \mathrm{p}<.05$, but no Treatment by Replication interaction. The significant replication effect was attributed to differences in animal shipments and laboratory conditions at the times the different replications were performed; however, given the lack of a Treatment by Replication interaction, the differences in replications were not deemed relevant to the interpretation of the treatments effect. Individual between-groups $t$ tests indicated that Groups ACQ and REM did not differ from each other, but that each differed significantly, ps $<.001$, from both Groups AMN and NC, which did not differ among themselves.

Examining the Day 4 scores of the AMN animals, "complete" amnesia was seen in all but one animal happening to belong to Replication 2, i.e., all other animals were indistinguishable from the average NC animal. Within-subject $t$ tests between Day 4 and Day 6 proved significant for the AMN subgroup that was shocked on Day $5, p<.05$, but not for the AMN subgroup that received no Day 5 treatment, $\mathrm{p}>.50$. A between-groups $t$ test for the two AMN subgroups tested on Day 6 also proved significant, $\mathrm{p}<.05$.

Although our focal data were reciprocal latencies, an analysis of hurdle-jumping frequencies was also performed. On Day 4, the ACQ, REM, NC, and AMN animals failed to hurdle-jump on a mean of $0.3 \%, 0.6 \%, 26.7 \%$, and $19.1 \%$ of their trials, respectively. The ACQ and REM groups differed significantly ( $\mathrm{ps}<.05$ ) from the $\mathrm{NC}$ and AMN groups on this measure, whereas no other differences were significant. Almost all of the failures to hurdle-jump in the AMN and NC groups occurred during the last half of the 25-trial blocks. These results are entirely consistent with those produced by the analyses of reciprocal latencies. Those animals in the AMN subgroup reminded on Day 5 and tested on Day 6 failed to hurdle-jump on a mean of $5.0 \%$ of their trials and did not differ significantly (ps $>.50$ ) from the performance of the ACQ and REM groups on Day 4.

\section{DISCUSSION}

On Day 4, evidence of amnesia for classically conditioned associations between the white compartment apparatus cues and FS is indicated by the low mean reciprocal latency of the AMN animals. This value is indistinguishable from that of animals run in pilot studies that received neither FS nor ECS. Evidence that this low mean is the result of ECS-induced amnesia rather than a lack of initial conditioning on Day 2 is provided by the high reciprocal latencies of the acquisition (ACQ) animals. The effectiveness of the noncontingent "reminder" FS can be seen in that the REM animals closely resemble those of Group ACQ and are quite dissimilar from the
AMN subjects. Finally, the possibility that the high reciprocal latencies of the REM animals are due to an interaction of Day 2 FS and ECS with Day 3 "reminder" FS producing changes in such nonassociative factors as increased overall activity level can be discounted by examination of the NC animals which received identical stimulation on Days 2 and 3, but received the Day 2 FS in the absence of the shuttlebox apparatus cues.

Our interpretation of the Day 4 data is predicated on the assumption that the REM animals were amnesic prior to "reminder" FS on Day 3; the basis for this, of course, is the performance of the AMN animals on Day 4. However, the possibility still remains that the REM animals were never amnesic or perhaps only some of them were amnesic, and the "reminder" FS of Day 3 merely enhanced the already present classically conditioned associations in the nonamnesic REM animals. The within-subjects aspect of this experiment was included to control for these possibilities. Examination of the Day 4 scores of each of the animals in the two AMN subgroups run on Days 5 and 6 indicate that on Day 4 these animals were indistinguishable from the average $\mathrm{NC}$ animal or, for that matter, from animals run in pilot studies that never received any treatment. Yet, "reminder" FS on Day 5 greatly enhanced the performance of these "certified fully amnesic animals." Herz and Peeke (1968) among others have suggested that repeated test trials can alter behavior even in the absence of memory of acquisition. However, examination of the Day 6 retest scores of the AMN subgroup that did not receive "reminder" FS on Day 5 indicates that this effect of repeated test trials in this particular situation is insignificantly small or nonexistent.

In summary, the present data demonstrate that, under conditions in which ECS will produce amnesia for classical conditioning, recovery of memory is still possible. Clearly, the hypothesis of Schneider et al. (1974) is not applicable to the present experiment. This suggests that the retrieval-failure explanation of ECS-induced amnesia is still the most comprehensive view proposed to date.

\section{REFERENCES}

Gold, P. E., \& KIng, R. A. Retrograde amnesia: Storage failure vs. retrieval failure. Psychological Review, 1974, 81, 465-469.

Herz, M. J., \& Peeke, H. V. S. ECS-produced retrograde amnesia: Permanence vs. recovery over repeated testing. Physiology and Behavior, 1968, 3, 517-521.

McAllister, D. E., \& McAllister, W. R. Forgetting of acquired fear. Journal of Comparative and Physiological Psychology, 1968, 65, 352-355.

McGaugh, J. L. Time dependent processes in memory storage. Science, 1966, 153, 1351-1358.

Miller, R. R., Ott, C. A., Berk, A. M., \& Springer, A. D. Appetitive memory restoration after electroconvulsive 
shock in the rat. Journal of Comparative and Physiological Psychology, 1974, 87, 717-723.

Miller, R. R., \& SPRINGER, A. D. Induced recovery of memory in rats following electroconvulsive shock. Physiology and Behavior, 1972, 8, 645-651.

Miller, R. R., \& Springer, A. D. Amnesia, consolidation, and retrieval. Psychological Review, 1973, 80, 69-79.

MilleR, R. R., \& SPRINGer, A. D. Implications of recovery from experimental amnesia. Psychological Review, 1974, 81, 470-473.

SChNeIDer, A. M., TAYLOR, J., \& Jinich, D. Recovery from retrograde amnesia: A learning process. Science, 1974, 184, 87-88.
SPRINGER, A. D. Vulnerability of skeletal and autonomic manifestations of memory in the rat to electroconvulsive shock. Journal of Comparative and Physiological Psychology, 1975, 88. 890-903.
(Received for publication April 29, 1975; revision accepted July 14, 1975.) 